\title{
HIGH-DIMENSIONAL OPTIMIZATION OF COLOR CODED APERTURES FOR COMPRESSIVE SPECTRAL CAMERAS
}

\author{
Hoover Rueda , Henry Arguello ${ }^{\dagger}$, and Gonzalo R. Arce \\ * Department of Electrical and Computer Engineering, University of Delaware, Newark, DE, USA. \\ ${ }^{\dagger}$ Department of Computer Science, Universidad Industrial de Santander, Colombia.
}

\begin{abstract}
A spectral image can be regarded as a three-dimensional cube where each pixel is a vector of intensities representing a spectral signature. Compressive spectral imaging (CSI) is a sensing and reconstruction framework, based on the fundamentals of the compressive sensing theory, which focuses on capturing spectral images efficiently, exploiting their highly correlated information by coding its spectral characteristics commonly using a black-and-white, grayscale or recently a color coded aperture. The distribution of the entries of the coded apertures determines the quality of the estimated spectral images. State of the art methods have used random coded apertures, and some optimization procedures have focused on the optimal design of horizontal sections of the coded apertures; however, they do not fully exploit the spatio-spectral correlations within the spectral images. To that end, in this paper, it is proposed a high-dimensional optimization procedure to design color coded apertures for CSI systems, which exploits not only the spectral correlations but also the spatial correlations within an spectral image. Simulations analyzing the conditioning of the sensing matrices, as well as the reconstruction quality of the attained spectral images show the improvement entailed by the proposed method.
\end{abstract}

Index Terms - Compressive spectral imaging, coded aperture design, color filter array, numerical optimization.

\section{INTRODUCTION}

Spectral imaging techniques captures sequences of twodimensional images along the electromagnetic spectrum, where each pixel is a vector of intensities representing a spectral reflectance signature. Information of the spectral properties of an object permits to know its components, which in turn can be used to detect or classify the elements in a certain image. As a result of the appearance of compressive sensing (CS) [1], new methodologies were proposed to effectively capture spectral images, such as compressive spectral imaging (CSI) [2]. CSI imaging systems are modeled based on the principles of a traditional spectrophotometer which records

This research was supported in part by Intel/NSF VEC 1538950. Hoover Rueda is supported by a Colciencias-Fulbright scholarship. all wavelengths simultaneously. Particularly, a ray of light emanating from the scene is passed through a series of optical elements, including apertures, gratings or prisms. Given the nature of the aperture, it can be designed to allow multiple rays of light to simultaneously impact onto the sensor. An arrangement of 2D spatially located apertures, is widely known as a coded aperture, where each pixel is a black, or translucent element in the conventional setting [3], or a tiny optical filter in recent developments [4]. Both the selection of the spectral characteristics of each pixel, as well as the spatial distribution of them within the 2D arrangement determine the quality of the spectral image to be estimated. Regarding the spectral characteristics, the use of optical filter-based coded apertures, so-called color coded apertures [4, 5], entails a single-step 3D spatial-spectral coding on the input data cube, compared to black-and-white or grayscale coded apertures, which only carry-out 2D spatial coding. Thus, the color coded aperture provides higher flexibility on the selection of voxels from the scene being integrated on the sensor.

Optimization of black-and-white coded apertures has been demonstrated in the past [6]. Regarding color coded apertures, a single work have reported their optimization [4], but focusing only on row-wise optimization, which does not fully exploit the spatial correlation within spectral images. To that end, in this paper, it is proposed a high-dimensional optimization procedure to design color coded apertures for CSI systems, exploiting not only the spectral correlations but also the 2D spatial correlations within an spectral image. The high-dimensional optimization focus on the design and selection of each element in the coded aperture, including the cut-off frequencies of the optical filters, as well as the exploitation of the correlation between rows, columns and spectral planes of the scene.

\section{COMPRESIVE SPECTRAL IMAGING SYSTEMS}

\subsection{CSI Sensing Model}

Different CSI architectures have been proposed to date, most of them using black-and-white coded apertures or spatial light modulators (SLMs) [3, 7], and just recent works use color coded apertures to perform the coding $[4,5,8]$. Prisms and gratings are conventionally used to disperse the optical field from the scene, and monochrome sensors are used to inte- 


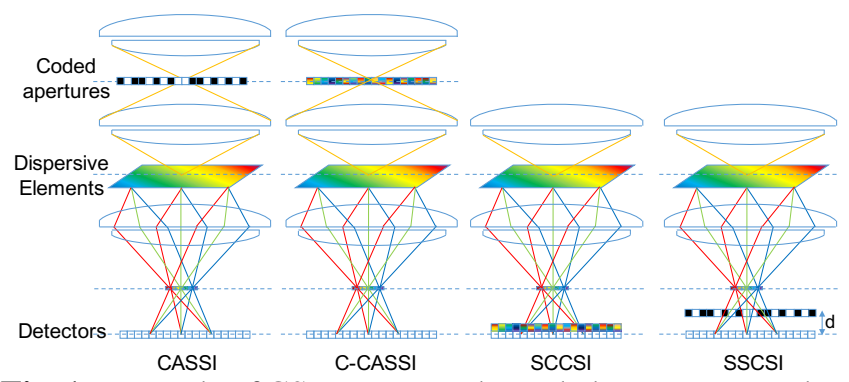

Fig. 1. Example of CSI systems: The coded aperture snapshot spectral imager (CASSI [3]), the CASSI with color-coded apertures (C-CASSI [5]), the snapshot colored compressive spectral imager (SCCSI [8]), and the spatial-spectral encoded compressive spectral imager (SSCSI [7]).

grate the modulated field. Figure 1 shows 4 examples of CSIs, widely known and used nowadays. For the scope of this paper, we focus on the single-disperser coded aperture snapshot spectral imager, which uses a prism and has been demonstrated using black-and-white (CASSI) and color coded apertures (C-CASSI). The use of color coded apertures provides improved results compared with black-and-white apertures [4], therefore we focus on the optimization of color coded apertures.

Let denote the spectral scene as the 3D function $f(x, y, \lambda)$, and the color coded aperture as the 3D function $T(x, y, \lambda)$, where $x, y$ represent the spatial coordinates and $\lambda$ the spectral domain. In particular the coded aperture function is given by,

$$
T^{\ell}(x, y, \lambda)=\sum_{n, m, k} t_{n, m, k}^{\ell} \operatorname{rect}\left(\frac{x}{\Delta_{c}}-m, \frac{y}{\Delta_{c}}-n, \frac{\lambda}{\Delta_{L}}-k\right),
$$

where $\Delta_{c}$ is the pitch size of the coded aperture, $\Delta_{L}$ represents the bandwidth of the color filters, $t_{n, m, k}^{\ell}$ is the coding applied by the $\ell^{t h}$ snapshot to the $(n, m, k)^{t h}$ voxel of the $Q=N \times N \times L$ spectral image, such that $n, m=$ $0,1, \ldots, N^{2}-1$ index the discretized spatial coordinates, and $k=0,1, \ldots, L-1$ the discrete spectral bands. Remark that $\ell=0,1, K-1$ accounts for the number of snapshots being captured, where the coded aperture pattern changes to capture new information from the scene. Assuming ideal spaceinvariant optical impulse response of the CSI system, the $\ell^{t h}$ compressive snapshot can be modeled as,

$$
g^{\ell}(x, y)=\int_{\Lambda} T^{\ell}(x+s(\lambda), y, \lambda) f_{0}(x+s(\lambda), y, \lambda) d \lambda,
$$

where $s(\lambda)$ represent the dispersion fuction of the prism, and $\Lambda$ is the spectral range sensitivity of the monochrome sensor.

\subsection{Higher-order discrete model approximation}

The bandwidth of each spectral band of the datacube is given by how much of the dispersion spread impinges onto a pixel of the sensor. This can be modeled as, $s\left(\lambda_{k}\right)-s\left(\lambda_{k+1}\right)=$ $k \Delta$, where $\Delta$ is the pitch size of the sensor. Figure 2 shows the propagation of a single voxel of the datacube through the

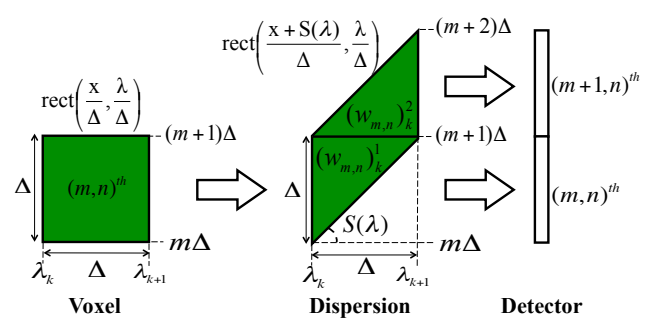

Fig. 2. Propagation of a single voxel through the CSI system.

optical system. There, it can be seen that the single voxel spreads onto more than a single FPA pixel due to the continuous nature of the dispersion function $s(\lambda)$ [9]. Mathematically, the portion of the voxel that impinges in each FPA pixel can be written as,

$$
\begin{gathered}
\left(w_{n, m}\right)_{k}^{r}=\iint_{n \Delta ; m \Delta ; \lambda_{k}}^{(n+1) \Delta ;(m+1) \Delta ; \lambda_{k+1}} \operatorname{rect}\left(\frac{x+s(\lambda)}{\Delta}-m^{\prime}, \frac{y}{\Delta}-n, \frac{\lambda}{\Delta}-k\right) \\
\quad \times \operatorname{rect}\left(\frac{x}{\Delta}-m, \frac{y}{\Delta}-n\right) d x d y d \lambda
\end{gathered}
$$

Therefore, Eq. (2) can be expressed in discrete form as

$$
g_{n, m}^{\ell}=\sum_{r} \sum_{k} t_{n, m-k-r, k}^{\ell} f_{n, m-k-r, k}\left(w_{n, m}\right)_{k}^{r} .
$$

\subsection{Matrix linear system model}

Expanding (4) to account for the full resolution of the sensor can be parsed as the linear system, $\mathbf{g}^{\ell}=\mathbf{H}^{\ell} \mathbf{f}$, where $\mathbf{f}_{q}=f_{\left(q-\left\lfloor\frac{q}{N}\right\rfloor N\right),\left(\left\lfloor\frac{q}{N}\right\rfloor-\left\lfloor\frac{q}{N^{2}}\right\rfloor N\right),\left\lfloor\frac{q}{N^{2}}\right\rfloor}$, for $q=0,1, \ldots, Q-1$. Note that this linear system, is highly under-determined, and exhibits a compression ratio of $R=V / Q \in[0,1]$, where 1 means no compression. Therefore, we need to force the sensing matrix to exhibit a close-to-well-conditioning behavior in order to obtain a good estimation of $\mathbf{f}$. The goal is to express the sensing matrix in terms of the entries of the coded aperture, such that a correct design of the latter entails a well conditioning of the former. The entries of the sensing matrix $\mathbf{H}^{\ell}$ are given by,

$$
\left(\mathbf{h}_{q}^{\ell}\right)_{v}=\sum_{q^{\prime}=0}^{Q-1}\left(\mathbf{p}_{q^{\prime}}\right)_{v}\left(\tilde{\mathbf{t}}_{q}^{\ell}\right)_{q^{\prime}}
$$

for $v=0,1, \ldots, V-1$, where $v$ and $q$ index the rows and columns, respectively. Note that the entries of $\mathbf{H}^{\ell}$ are the inner products between two matrices $\mathbf{H}^{\ell}=\mathbf{P} \mathbf{T}^{\ell}$, such that $\mathbf{T}^{\ell}=\left[\tilde{\mathbf{t}}_{0}^{\ell}, \tilde{\mathbf{t}}_{1}^{\ell}, \ldots, \tilde{\mathbf{t}}_{Q-1}^{\ell}\right]$ represents the effect of the coded aperture, and $\mathbf{P}=\left[\mathbf{p}_{0}, \mathbf{p}_{1}, \ldots, \mathbf{p}_{Q-1}\right]$, represents the effect of the dispersive element, with $\tilde{\mathbf{t}}_{q}^{\ell} \in \mathbb{R}^{Q}$ and $\mathbf{p}_{q} \in \mathbb{R}^{V}$. The entries of this matrices are given by,

$$
\left(\tilde{\mathbf{t}}_{q}^{\ell}\right)_{q^{\prime}}=\left\{\begin{array}{cc}
\left(\mathbf{t}_{\left\lfloor\left\lfloor\frac{q}{N^{2}}\right\rfloor\right.}^{\ell}\right)_{q^{\prime}-\left\lfloor\frac{q^{\prime}}{N^{2}}\right\rfloor N^{2}}, & \text { if } q=q^{\prime} \\
0, & \text { otherwise }
\end{array},\right.
$$

such that $\left(\mathbf{t}^{\ell}\right)_{q}=t_{\left(q-\left\lfloor\frac{q}{N}\right\rfloor N\right),\left(\left\lfloor\frac{q}{N}\right\rfloor-\left\lfloor\frac{q}{N^{2}}\right\rfloor N\right),\left\lfloor\frac{q}{N^{2}}\right\rfloor}^{\ell}$, and, 


$$
\left(\mathbf{p}_{q^{\prime}}\right)_{v}=\left\{\begin{array}{cc}
\left(\mathbf{w}^{r}\left\lfloor\frac{q^{\prime}}{N^{2}}\right\rfloor\right)_{q^{\prime}-\left\lfloor\frac{q^{\prime}}{N^{2}}\right\rfloor N^{2}}, & \text { if } v=q^{\prime}-q_{r}^{\prime \prime} \\
0, & \text { otherwise }
\end{array}\right.
$$

such that $\left(\mathbf{w}_{k}^{r}\right)_{q}=\left(w_{\left(q-\left\lfloor\frac{q}{N}\right\rfloor N\right),\left(\left\lfloor\frac{q}{N}\right\rfloor-\left\lfloor\frac{q}{N^{2}}\right\rfloor N\right)}\right)_{k}^{r}$, and $q_{r}^{\prime \prime}=$ $\left\lfloor\frac{q^{\prime}}{N^{2}}\right\rfloor N^{2}+r N$. Evaluating the summation in Eq. (5) in terms of Eqs. (6) and (7), and noting that Eq. (6) describes the entries of a block-diagonal matrix, then it is obtained,

$$
\left(\mathbf{h}_{q}^{\ell}\right)_{v}=\left\{\begin{array}{cl}
\left(\mathbf{w}^{r}\left\lfloor\frac{q}{N^{2}}\right\rfloor\right)_{q-\left\lfloor\frac{q}{N^{2}}\right\rfloor N^{2}}\left(\mathbf{t}_{\left\lfloor\frac{q}{N^{2}}\right\rfloor}^{\ell}\right)_{q-\left\lfloor\frac{q}{N^{2}}\right\rfloor N^{2}}, & \text { if } v=g_{r} \\
0, & \text { otherwise }
\end{array}\right.
$$

where $g_{r}=q-\left\lfloor\frac{q}{N^{2}}\right\rfloor N^{2}+r N$, for $r=0,1, \ldots, R-1$.

\section{OPTIMIZATION MODEL}

\subsection{CS inverse problem estimation}

Note that in order to obtain a reconstruction from a underdetermined linear system of equations, certain conditions must be imposed such that the recovery becomes feasible. The theory of CS has emerged to solve this kind of problems in a reliable way. One of the general conditions CS proposes is to impose that the input data can be represented in a very compact way (sparse) when projected over an orthogonal basis. This can be mathematically modeled as,

$$
\mathbf{g}=\mathbf{H f}=\mathbf{H} \Psi \boldsymbol{\theta}=\mathbf{A} \boldsymbol{\theta}
$$

where $\theta \in \mathbb{R}^{Q}$ is a compact (or sparse) version of the signal $\mathbf{f}$ in the basis $\Psi \in \mathbb{R}^{Q \times Q}, \mathbf{H}=\left[\left(\mathbf{H}^{0}\right)^{T}, \ldots,\left(\mathbf{H}^{K-1}\right)^{T}\right]^{T}$ is the multishot sensing matrix, and $\mathbf{g}=\left[\left(\mathbf{g}^{0}\right)^{T}, \ldots,\left(\mathbf{g}^{K-1}\right)^{T}\right]^{T}$ is the resulting set of measurements.

The vector $\boldsymbol{\theta}$ is said to be a compact representation of $\mathbf{f}$ because all the information of the latter is concentrated in just few coefficients of the former. That is, $\|\operatorname{supp}(\boldsymbol{\theta})\|=\|\{j$ : $\left.\left|\boldsymbol{\theta}_{j}\right|>0\right\} \|=S$, with $S<<Q$. By multiplying the representation domain $\Psi$ with the transfer function $\mathbf{H}$, it is obtained the compressive sensing matrix $\mathbf{A} \in \mathbb{R}^{K V \times Q}$. Based on the sparse assumption, CS permits to recover an estimation of the data cube $\hat{\mathbf{f}}$ from the compressive measurements $\mathbf{g}$ by solving the LASSO-related minimization problem,

$$
\hat{\mathbf{f}}=\boldsymbol{\Psi}^{-\mathbf{1}}\left(\operatorname{argmin}_{\boldsymbol{\theta}^{\prime}}\left\|\mathbf{g}-\mathbf{A} \boldsymbol{\theta}^{\prime}\right\|_{2}^{2}+\tau\left\|\boldsymbol{\theta}^{\prime}\right\|_{1}\right),
$$

with $\tau>0$ being a regularization parameter.

\subsection{Design of the sensing operator $A$}

Compressive sensing also requires that the matrix A satisfy certain condition, such as the restricted isometry property (RIP), or similarly, that the matrices $\mathbf{H}$ and $\boldsymbol{\Psi}$ be mutually incoherent [4]. Satisfying these conditions guarantees, with high probability, the correct estimation of the input signal. In particular, the RIP for the matrix $\mathbf{A}$ of order $S$ is defined as the smallest $\delta_{S}$ such that,

$$
\left(1-\delta_{S}\right)\|\boldsymbol{\theta}\|_{2}^{2} \leq\|\mathbf{A} \boldsymbol{\theta}\|_{2}^{2} \leq\left(1+\delta_{S}\right)\|\boldsymbol{\theta}\|_{2}^{2},
$$

where $\delta_{S}$ is given by, $\max _{S \subset[Q],|\rho| \leq S}\left\|\mathbf{A}_{|\rho|}^{T} \mathbf{A}_{|\rho|}-\mathbb{I}\right\|_{2}^{2}$,

with $\mathbf{A}_{|\rho|}$ being a $K V \times|\rho|$ matrix whose columns are equal to $|\rho|$ columns of $\mathbf{A}$ indexed by the set $\rho$, and $\mathbb{I}$ is an identity matrix. To satisfy the RIP condition, the matrix $\mathbf{A}$ must be carefully designed. This matrix relies directly on the selection of the representation matrix $\Psi$ along with the structure of the sensing matrix $\mathbf{H}$. In particular, based on Eq. (8), the entries of $\mathbf{A}=\mathbf{H} \Psi=\left[\mathbf{a}_{0}, \mathbf{a}_{1}, \ldots, \mathbf{a}_{Q-1}\right]$, where $\Psi=\left[\begin{array}{llll}\boldsymbol{\psi}_{0} & \boldsymbol{\psi}_{1} \ldots \boldsymbol{\psi}_{Q-1}\end{array}\right]$, are given by,

$$
\begin{gathered}
\left(\mathbf{a}_{j^{\prime}}\right)_{i^{\prime}}=\sum_{r=0}^{R-1} \sum_{k=0}^{L-1}\left(\mathbf{w}_{k}^{r}\right)_{i^{\prime}-(k+r) N-\left\lfloor\frac{i^{\prime}}{V}\right\rfloor V}\left(\mathbf{t}_{k}^{\left\lfloor\frac{i^{\prime}}{V}\right\rfloor}\right)_{i^{\prime}-(k+r) N-\left\lfloor\frac{i^{\prime}}{V}\right\rfloor V} \\
\times\left(\boldsymbol{\psi}_{j^{\prime}}\right)_{i^{\prime}-(k+r) N-\left\lfloor\frac{i^{\prime}}{V}\right\rfloor V+k N^{2}},
\end{gathered}
$$

for $i^{\prime}=0, \ldots, K V-1$, and $j^{\prime}=0, \ldots, Q-1$. Defining the matrix $\mathbf{A}_{|\rho||\rho|}=\mathbf{A}_{|\rho|}^{T} \mathbf{A}_{|\rho|}, \delta_{S}$ can be rewritten as

$$
\delta_{S}=\max _{\rho \subset[Q],|\rho| \leq S} \lambda_{\max }\left(\mathbf{A}_{|\rho||\rho|}-\mathbf{I}\right) \text {, }
$$

where $\mathbf{A}_{|\rho||\rho|}$ is given by,

$$
\begin{aligned}
\sum_{\ell=0}^{K-1} & \sum_{i=0}^{V-1} \sum_{r_{1}=0}^{R-1} \sum_{k_{1}=0}^{L-1} \sum_{r_{2}=0}^{R-1} \sum_{k_{2}=0}^{L-1}\left(\mathbf{w}_{k_{1}}^{r_{1}}\right)_{i-\left(k_{1}+r_{1}\right) N}\left(\mathbf{w}_{k_{2}}^{r_{2}}\right)_{i-\left(k_{2}+r_{2}\right) N} \\
& \times\left(\mathbf{t}_{k_{1}}^{\ell}\right)_{i-\left(k_{1}+r_{1}\right) N}\left(\mathbf{t}_{k_{2}}^{\ell}\right)_{i-\left(k_{2}+r_{2}\right) N} \\
& \times\left(\boldsymbol{\psi}_{l_{1}}\right)_{i-\left(k_{1}+r_{1}\right) N+k_{1} N^{2}}\left(\boldsymbol{\psi}_{l_{2}}\right)_{i-\left(k_{2}+r_{2}\right) N+k_{2} N^{2}}
\end{aligned}
$$

\subsection{Proposed optimization model}

Given that the weights $\mathbf{w}_{k}^{r}$ and the basis $\Psi$ are fixed and bounded, the first depends on the dispersive element, and the second on a-priori selection, the entries of $\mathbf{A}_{|\rho||\rho|}$ in Eq. (15) can be modeled as a sub-Gaussian random variable, such that the minimization of its parameter $\beta=$

$$
\sum_{\ell=0}^{K-1} \sum_{i=0}^{V-1} \sum_{r_{1}=0}^{R-1} \sum_{k_{1}=0}^{L-1} \sum_{r_{2}=0}^{R-1} \sum_{k_{2}=0}^{L-1}\left(\mathbf{t}_{k_{1}}^{\ell}\right)_{i-\left(k_{1}+r_{1}\right) N}\left(\mathbf{t}_{k_{2}}^{\ell}\right)_{i-\left(k_{2}+r_{2}\right) N}
$$

yields to a small parameter $\delta_{s}$, and so, to better satisfy the RIP. Note that $\beta$ can be minimized by minimizing the products of the entries of the coded aperture $\left(\mathbf{t}_{k_{1}}^{\ell}\right)_{i-\left(k_{1}+r_{1}\right) N} \times$ $\left(\mathbf{t}_{k_{2}}^{\ell}\right)_{i-\left(k_{2}+r_{2}\right) N}$. This problem can be cast as the minimization of the products of the entries of the coded aperture within a window of size $R+L \times R+L$, around each pixel ( $i-$ $\left.\left(k_{1}+r_{1}\right) N\right)$ of each band $k_{1}$, for every $\ell^{t h}$ snapshot being captured. Intuitively, we seek to spread the number of translucent elements of the coded aperture within a cube of size $R+L \times R+L \times L$ centered at the pixel $\left(i-\left(k_{1}+r_{1}\right) N\right)$. Formally, this can be written as the optimization problem

$$
\begin{aligned}
& \underset{\left\{\mathbf{t}_{0}^{\ell}, \ldots, \mathbf{t}_{L-1}^{\ell}\right\}_{\ell=0}^{K-1}}{\operatorname{argmin}} \sum_{k=1}^{L} \sum_{i=1}^{R+L} \sum_{j=1}^{R+L} \tau_{k} U_{i, j}+\tau_{i} V_{j, k}+\tau_{j} W_{i, k} \\
& \text { subject to } \sum_{\ell=0}^{K-1} \mathbf{t}_{k}^{\ell}=1, \text { for } k=0, \ldots, L-1
\end{aligned}
$$


where, $U_{i, j}$ controls the spread in the $x-y$ range of the cube, $V_{j, k}$ controls the spread along the $y-\lambda$ domain, $W_{i, k}$ controls the $x-\lambda$ spread, and $\tau_{k}, \tau_{i}, \tau_{j}$ are weight constants, which prioritize one kind of spreading over the others. Note that, the constraint guarantees obtaining a solution different from the trivial (all-zero) coded aperture, which optimally minimizes Eq. (16). To solve the optimization problem proposed in Eq. (17), we developed an iterative algorithm which randomly walks along all the pixels, of a set of randomly generated coded apertures satisfying the complementary constraint, and evaluates the objective function of the problem for each snapshot. The snapshot pixel that gives the lowest objective value is set to 1 , and the rest are set to 0 . The algorithm iterates on the updated coded aperture until the objective function stops decreasing.

\section{SIMULATIONS}

\subsection{Coded aperture optimization results}

To evaluate the outcome of the proposed method, we perform a comparison against random coded apertures, and against the coded apertures resulting of the row-wise optimization algorithm [4]. A widely use method to compare different measurement strategies is the singular value decomposition (SVD) analysis of the sensing matrices $\mathbf{A}$ from each method [10]. This analysis does not depend on prior information about the scene, and it relies on the condition number $(\kappa)$, defined as the ratio of the greatest singular value to the least nonzero singular value, to measure how ill-conditioned is the problem; the closer the condition number to 1 , the better conditioned the matrix. Figure 3 shows the SVD curves of the 3 methods being compared. It can be seen that the proposed method attain the best condition number for the first 50 and 100 components, and exhibits a slower-decaying behavior of the SVD coefficients, thus it is prone to capture more orthogonal components of the scene.

A single color-coded aperture realization of each of the three methods being compared is depicted in Fig. 4. Recall that the color-coded aperture can be regarded as a 3D cube, thus a front view $(x-y)$ and a top view $(x-\lambda)$ are shown. It can be seen that the row-wise optimization method exhibits an structured pseudo-random behavior due to its row-wise optimization nature, whereas the proposed method spreads the non-zero values along the 3 dimensions.
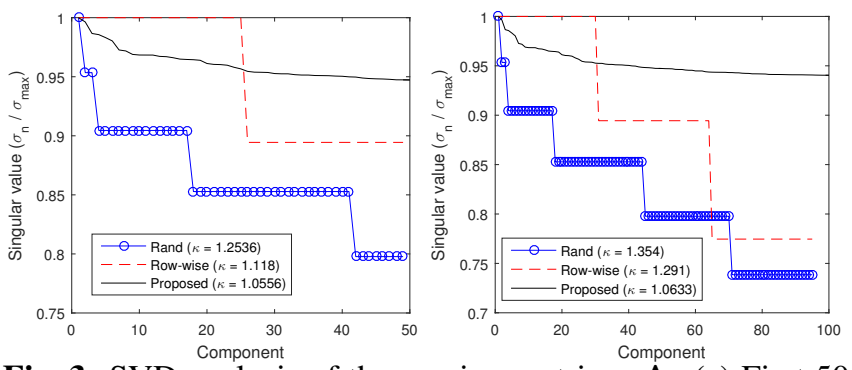

Fig. 3. SVD analysis of the sensing matrices A. (a) First 50 components, (b) First 100 components.

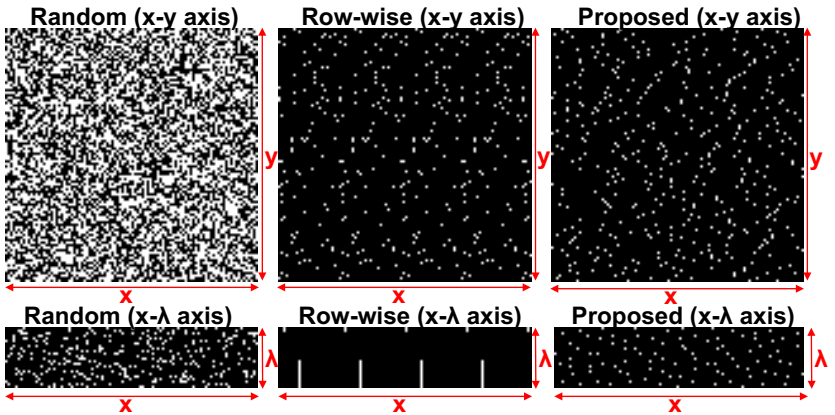

Fig. 4. Comparison of colored coded apertures. (First row) Front view, and (Second row) Top view.

\subsection{Testing optimized system reconstruction}

To test the impact of the proposed coded aperture optimization algorithm in the quality of the reconstruction of spectral images, we use 2 spectral data cubes (Balloons and Beads) with $N \times N=256 \times 256$ pixels of spatial resolution, and $L=24$ spectral bands, from the CAVE database [11]. We tested different scenarios using different amounts of snapshots $K=2,4, \ldots, 24$, and the three kinds of coded apertures. The reconstruction were attained by solving the CS problem in Eq. (10), employing the gradient projection for sparse reconstruction (GPSR) algorithm [12], using the Kronecker product between the 2D Wavelet Symmlet 8 transform and the 1D discrete cosine transform, as the representation basis function $\Psi$. This configuration and this algorithm have shown to be reliable and relatively fast to attain good reconstructions. The best penalization parameter $\tau$ was found by try and error within the range $\tau \in[3 e-6,3 e-4]$ for each case. Figure 5 summarizes the reconstruction results in terms of the peak signal to noise ratio (PSNR) and the structural similarity index (SSIM). Remark that the larger the PSNR the better the reconstruction, and similarly, the closest the SSIM index to 1 , the better the reconstruction. Therefore, it can be
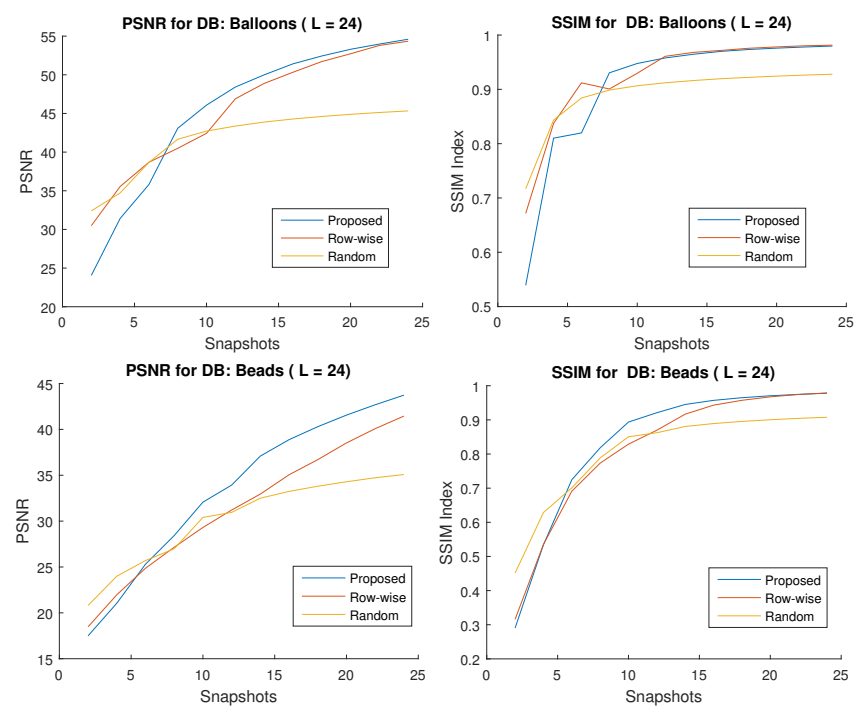

Fig. 5. Overall reconstruction comparison in terms of PSNR and SSIM for different snapshots using the three methods. 


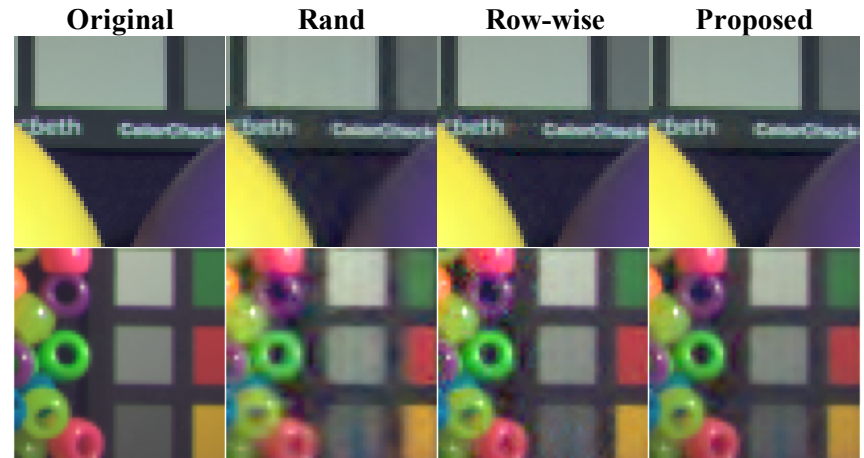

Fig. 6. Zoom sections of the reconstructions mapped to RGB color-space, when (First row) $K=12$ are captured for the balloons. (Second row) $K=10$ are captured for the beads.

noticed that the proposed optimization algorithm generates better coded apertures for the CSI system, as its PSNR and SSIM overcomes the row-wise and the randomly generated. It worth pointing that the proposed algorithm perform better for larger number of snapshots since the number of non-zero values, per snapshot, decreases to satisfy the complementary constraint in Eq. (17). Figure 6 shows a comparison of the estimated spectral images when $K=12$ snapshots are captured by the CSI system for the Balloons, and $K=10$ for the Beads. The cumulative errors along the 24 spectral bands are shown for ease of interpretation in Fig. 7. Finally, Fig. 8 shows 3 out of the 24 spectral bands of Fig. 6(b), to see the improvement at wavelength level. Although not easily noticeable, the images at the right-most column preserves better the edges, and they are more artifact-free.

\section{CONCLUSIONS}

A new optimization of colored coded apertures has been proposed and demonstrated. The attained optimal coded apertures overcomes the quality of up-to-date proposed coded aperture optimization procedures. In particular, the optimal coded apertures entail an improvement of around $8 \mathrm{dBs}$ in terms of PSNR against random distributions and $3 \mathrm{dBs}$

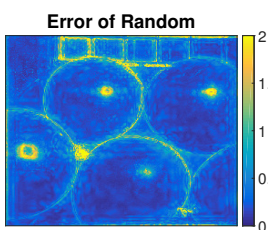

Error of Random

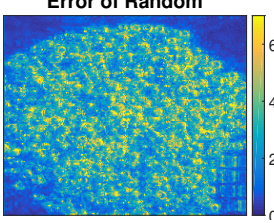

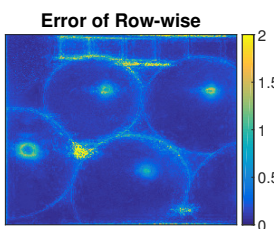

Error of Row-wise

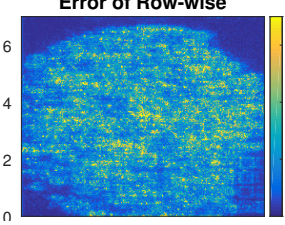

Error of Proposed

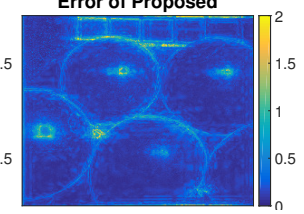

Error of Proposed

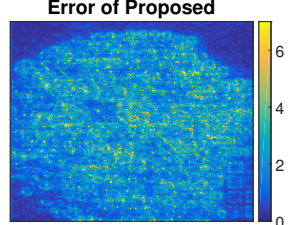

Fig. 7. Cumulative errors of the reconstructions for (First row) Balloons database with $K=12$, and (Second row) Beads database with $K=10$. (Units in \%)

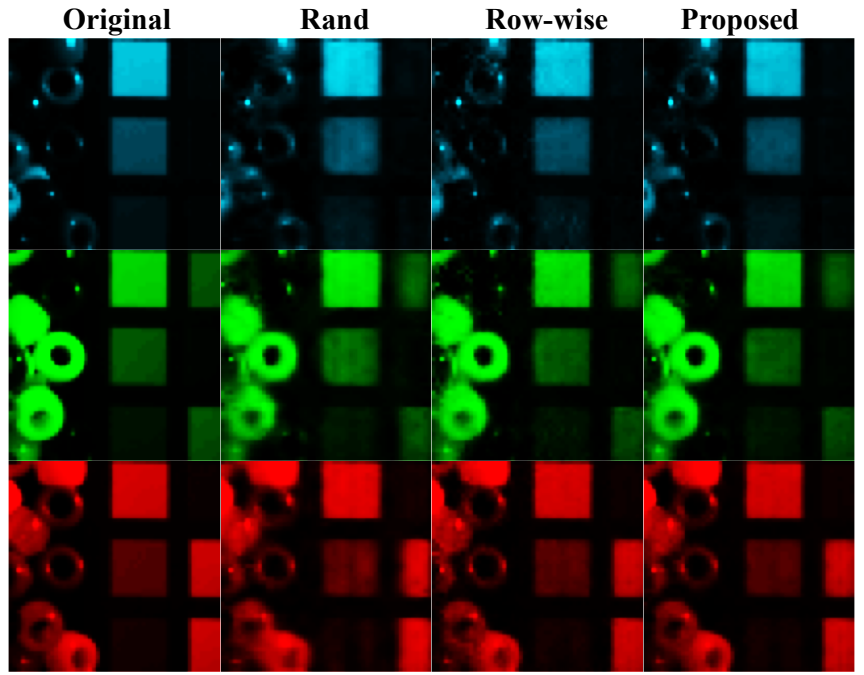

Fig. 8. Reconstructions results of the $5^{t h}, 10^{t h}$ and $20^{t h}$ spectral bands for the beads database, using $K=10$.

around row-wise optimization. Furthermore, the proposed coded apertures entail a better conditioned sensing matrix with a condition number of $\kappa=1.0633$ for the 100 first singular values.

\section{REFERENCES}

[1] E. J. Candes and M. B. Wakin, "An Introduction To Compressive Sampling," IEEE Signal Processing Magazine, vol. 25, no. 2, pp. 21-30, 2008.

[2] G. R. Arce, D. J. Brady, L. Carin, H. Arguello and D. S. Kittle, "An introduction to compressive coded aperture spectral imaging," IEEE Signal Processing Magazine, vol. 31, no. 1, pp. 105-115, 2014.

[3] D. Kittle, K. Choi, A. A. Wagadarikar and D. J. Brady, "Multiframe image estimation for coded aperture snapshot spectral imagers," Applied Optics, vol. 49, pp. 6824-6833, 2010.

[4] H. Arguello and G. R. Arce, "Colored coded aperture design by concentration of measure in compressive spectral imaging," IEEE Transactions on Image Processing, vol. 23, no. 4, pp. 1896-1908, 2014.

[5] H. Rueda, H. Arguello and G. R. Arce, "Compressive spectral testbed imaging system based on thin-film color-patterned filter arrays," Applied Optics, vol. 55 pp. 9584-9593, 2016.

[6] H. Arguello and G. R. Arce, "Rank minimization code aperture design for spectrally selective compressive imaging," IEEE Transactions on Image Processing, vol. 22, no. 3, pp. 941-954, 2013.

[7] X. Lin, Y. Liu, J. Wu and Q. Dai, "Spatial-spectral encoded compressive hyperspectral imaging", ACM Trans. Graph. (SIGGRAPH Asia), vol. 33, no. 6, pp. 233, 2014.

[8] C. V. Correa, H. Arguello and G. R. Arce, "Snapshot colored compressive spectral imager," J. Opt. Soc. Am. A, vol. 32, pp. 1754-1763, 2015.

[9] H. Arguello, H. Rueda, Y. Wu, D. W. Prather and G. R. Arce, "Higherorder computational model for coded aperture spectral imaging," Applied Optics, vol. 52, no. 10, pp. D12-D21, 2013.

[10] D. J. Brady, Optical Imaging and Spectroscopy, Wiley-Interscience, (2009).

[11] CAVE Multispectral database. Available: http://www.cs.columbia.edu/CAVE/databases/multispectral/

[12] M. A. T. Figueiredo, R. D. Nowak and S. J. Wright, "Gradient projection for sparse reconstruction: Application to compressed sensing and other inverse problems," IEEE Journal of Selected Topics in Signal Processing, vol. 1, no. 4, pp. 586-597, 2007. 